\title{
THE IONIZATION STRUCTURE OF PLANETARY NEBULAE
}

\author{
T. BARKER \\ Department of Physics and Astronomy, Wheaton College, Norton, MA 02766, USA
}

Ground-based and satellite spectrophotometric observations of emission-line intensities over the spectral range 1400-7200 $\AA$ have been made in 5 or more positions in a total of 9 planetary nebulae.

Since 1980, I have measured emission-line intensities over the spectral range $1400-7200 \AA$ in 5 or more positions in a total of 9 planetary nebulae (NGC 6720, NGC 7009, NGC 6853, NGC 3242, NGC 7662, NGC 6826, NGC 1535, NGC2392, and NGC 2440). The goals of these studies include: (1) to observe elements in more stages of ionization than is possible from optical spectra alone; this provides a check on optical ionization correction procedures, which are still needed for nebulae which are too faint to observe with the IUE satellite; (2) to get particularly accurate total abundances for each nebula by averaging measurements made in different nebular locations; such differences can be sensitive tests of theories of mixing of processed material in the planetary progenitors; (3) to test theoretical ionization models. In most nebulae, electron temperatures determined from different indicators such as $\mathrm{H}^{+}, \mathrm{O}^{+}, \mathrm{O}^{++}, \mathrm{S}^{+}$, and $\mathrm{S}^{++}$, and $\mathrm{Ne}^{3+}$ agree fairly closely, but for others, the more highly-ionized species indicate significantly higher temperatures. For all planetaries studied so far, optically-measured abundances agree well with UV determinations, with the exception of $\mathrm{C}^{++}$, where the optical measurement is systematically higher. Since this discrepancy is systematically greater closer to the central star, I believe that the $\lambda 4267 \mathrm{C}$ II line intensity is not being interpreted correctly, either because it is blended with another line, or because the excitation mechanism for the $\lambda 4267$ line is not fully understood. Standard ionization- correction equations give consistent results for the different positions which are in excellent agreement with abundances calculated using ultraviolet lines, and there is no evidence for abundance gradients in any of the nebulae. The elemental abundances do differ slightly between nebulae, implying differences both in the composition of the gas that formed the progenitor stars and differences in the amount of mixing of processed material in the stars before envelope ejection. Average logarithmic abundances (relative to $\mathrm{H}=12.00$ ) are: $\mathrm{He}=11.00, \mathrm{O}=8.74, \mathrm{~N}=8.12, \mathrm{Ne}=8.12, \mathrm{C}=8.67, \mathrm{Ar}=6.27$, and $\mathrm{S}=$ 6.82 . 